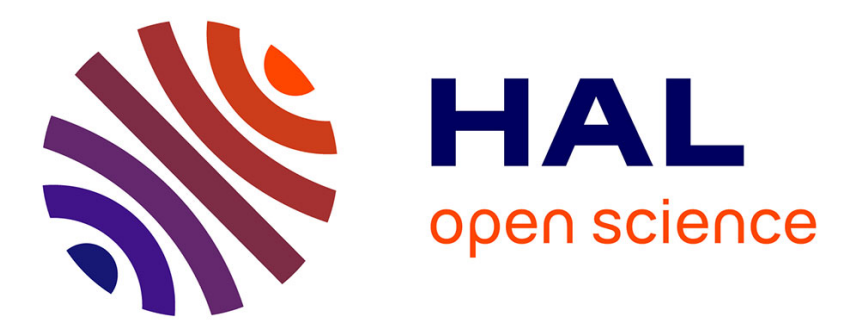

\title{
Short-Term Frequency Stability Measurement of BVA Oscillators
}

\author{
Jan Cermak, Alexander Kuna, Ludvík Sojdr, Patrice Salzenstein
}

\section{To cite this version:}

Jan Cermak, Alexander Kuna, Ludvík Sojdr, Patrice Salzenstein. Short-Term Frequency Stability Measurement of BVA Oscillators. IEEE Frequency Control Symposium \& European Frequency and Time Forum, Jun 2007, Geneva, Switzerland. pp.1255-1260, 10.1109/FREQ.2007.4319278 . hal00154292

\section{HAL Id: hal-00154292 \\ https://hal.science/hal-00154292}

Submitted on 13 Jun 2007

HAL is a multi-disciplinary open access archive for the deposit and dissemination of scientific research documents, whether they are published or not. The documents may come from teaching and research institutions in France or abroad, or from public or private research centers.
L'archive ouverte pluridisciplinaire HAL, est destinée au dépôt et à la diffusion de documents scientifiques de niveau recherche, publiés ou non, émanant des établissements d'enseignement et de recherche français ou étrangers, des laboratoires publics ou privés. 


\section{Short-Term Frequency Stability Measurement of BVA Oscillators}

\author{
Jan Čermák, Alexander Kuna, Ludvík Šojdr \\ Institute of Photonics and Electronics \\ Academy of Sciences of the Czech Republic \\ Prague, Czech Republic \\ cermak@ufe.cz
}

\author{
Patrice Salzenstein \\ FEMTO-ST Institute \\ CNRS
}

Besançon, France

patrice.salzenstein@1pmo.edu

\begin{abstract}
This paper discusses the time-domain measurement of short-term frequency stability of ultra-stable BVA oscillators with flicker frequency modulation noise on the order of $10^{-14}$ in terms of Allan deviation at averaging intervals from hundreds of milliseconds to tens of seconds. The stability has been measured with a highly sensitive phase-time comparator based on the dual-mixer time-difference multiplication with a background instability of $\sim 7 \times 10^{-15} / \tau$. A discrepancy has been observed in the comparator background noise found with two signals from a single oscillator (comparator test) and with two signals from two oscillators (stability measurement).
\end{abstract}

\section{INTRODUCTION}

The state-of-the-art BVA quartz oscillators [1] exhibit, besides a very good long-term frequency stability thanks to the BVA technique, an excellent short-term stability with a flicker frequency modulation (FFM) on the order of $10^{-14}$ in terms of Allan deviation in averaging intervals from hundreds of milliseconds to a few tens of seconds. To measure this ultra-low noise floor we need a highly sensitive measurement system in the time domain and also very good measurement conditions. In this paper we will discuss the problems associated with this challenging measurement which differs from common ones by three respects:

- The variations to be measured are comparable to variations originating from the measurement system.

- The compared signals have about the same stability so that none of them can be considered a reference.

- There are no signals to calibrate the measurement system because the best test signals available are the ones to be measured.
Our experience presented in this paper is based on the time-domain measurements of $5 \mathrm{MHz}$ Oscilloquartz (OSA) $8600 / 8607$ oscillators [2], [3] carried out at the Institute of Photonics and Electronics (IPE), former Institute of Radio Engineering and Electronics.

We refer to the measurements of four oscillators (two of IPE, one of FEMTO-ST and one of OSA) during a week-long measurement campaign in February 2006 and to a great number of repeated measurements of the two IPE oscillators performed at a later time.

\section{MEASUREMENT BASICS}

The measured quantity is the variations in the phase-time difference, $x_{v}(t)$, between two quasi-synchronous sine-wave signals at nearly equal frequency $v$. To enhance the measurement sensitivity, the variation $x_{\mathrm{v}}(t)$ is first magnified to $x(t)=M x_{\mathrm{v}}(t)$, where $M$ is the multiplication factor, using the dual-mixer time-difference multiplication (DMTDM). The DMTDM technique has been known for years [4] and it is still considered to be the best-suited technique for highly sensitive phase-time measurements [5], [6], [7], [8], [9], [10], [11], [12]. The method is based on dual mixing the two compared signals at $v$ with a signal at $v \pm v_{\mathrm{B}}$ from a common oscillator (CO) to provide two beat-note signals at $v_{B}$ as shown schematically in Fig.1. The multiplication factor is thus $M=v / v_{\mathrm{B}}$.

A phase-time comparator (time-interval counter) then samples the process $x(t)$ by periodically measuring the time interval between two adjacent zero-crossings of the compared signals, which occur at instances $t_{\mathrm{k}}$ and $t_{\mathrm{k}}+x_{\mathrm{k}}$, respectively. The measurement result is the frequency stability/instability (we will use these terms interchangeably) estimated from the sequence $\left\{x_{\mathrm{k}}\right\}$ in terms of Allan deviation $\sigma_{\mathrm{y}}(\tau)$ as a function of the averaging time interval $\tau$ [13], [14]. 


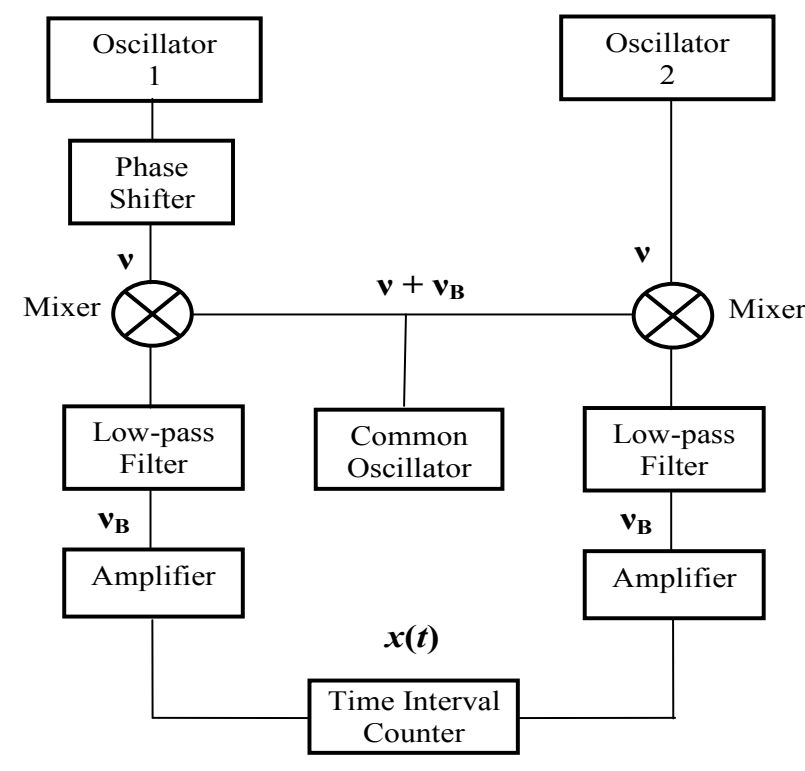

Figure 1. DMTDM setup.

In most of the stability analysis we have used the Stable32 software package [15] that provides the uncertainty of $\sigma_{\mathrm{y}}(\tau)$ estimates based on the Chi-squared distribution with the equivalent degrees of freedom for a given $\tau, N$ and the prevailing noise type [16]. If not mentioned otherwise, we use the $68.3 \%$ probability uncertainty throughout this text. The deviation $\sigma_{\mathrm{y}}(\tau)$ is the common overlapping Allan deviation estimated out of $N$ samples as

$$
\sigma_{\mathrm{y}}(\tau)=\sqrt{\left(\frac{1}{2(N-2 m) \tau^{2}} \sum_{i=1}^{N-2 m}\left(x_{\mathrm{i}+2 \mathrm{~m}}-2 x_{\mathrm{i}+\mathrm{m}}+x_{\mathrm{i}}\right)^{2}\right)}
$$

where $\tau=m \tau_{0}$ and $\tau_{0}$ is the basic sampling interval.

In modeling the variations involved in the measurement, we think it useful to introduce a concept of inherent variations as measured in near ideal conditions. Thus we presume that the oscillator pair has its inherent stability ${ }^{\mathrm{P}} \sigma_{y}(\tau)$, in terms of Allan deviation, and the comparator has its inherent stability ${ }^{\mathrm{C}} \sigma_{\mathrm{y}}(\tau)$. In real measurement conditions we have to take into account additional variations that cause the instability ${ }^{\mathrm{M}} \sigma_{\mathrm{y}}(\tau)$. These three contributions, assumed uncorrelated, give the total Allan deviation obtained from the measurement

$$
\sigma_{\mathrm{y}}(\tau)=\left[{ }^{\mathrm{P}} \sigma_{\mathrm{y}}(\tau)^{2}+{ }^{\mathrm{C}} \sigma_{\mathrm{y}}(\tau)^{2}+{ }^{\mathrm{M}} \sigma_{\mathrm{y}}(\tau)^{2}\right]^{1 / 2}
$$

Obviously, the minimum of (2) is

$$
\min \sigma_{\mathrm{y}}(\tau)=\left[{ }^{\mathrm{P}} \sigma_{\mathrm{y}}(\tau)^{2}+{ }^{\mathrm{C}} \sigma_{\mathrm{y}}(\tau)^{2}\right]^{1 / 2}
$$

The inherent pair stability ${ }^{\mathrm{P}} \sigma_{\mathrm{y}}(\tau)$ could be approximated by measuring the oscillators with a comparator that has
${ }^{\mathrm{C}} \sigma_{\mathrm{y}}(\tau)<{ }^{\mathrm{P}} \sigma_{\mathrm{y}}(\tau)$ in measurement conditions with ${ }^{\mathrm{M}} \sigma_{\mathrm{y}}(\tau)<<{ }^{\mathrm{P}} \sigma_{\mathrm{y}}(\tau)$. Similarly, the inherent comparator stability ${ }^{\mathrm{C}} \sigma_{\mathrm{y}}(\tau)$ would be found by testing the comparator with the signals having ${ }^{\mathrm{P}} \sigma_{\mathrm{y}}(\tau)<<{ }^{\mathrm{C}} \sigma_{\mathrm{y}}(\tau)$ in conditions with ${ }^{\mathrm{M}} \sigma_{\mathrm{y}}(\tau)<<{ }^{\mathrm{C}} \sigma_{\mathrm{y}}(\tau)$. Obviously, in a good measurement we should have $\sigma_{\mathrm{y}}(\tau) \sim{ }^{\mathrm{P}} \sigma_{\mathrm{y}}(\tau)$.

Presuming that the inherent stability ${ }^{\mathrm{P}} \sigma_{\mathrm{y}}(\tau)$ is given by pure power-law noises then ${ }^{\mathrm{P}} \sigma_{\mathrm{y}}(\tau)$ is well reproducible and the FFM floor of ${ }^{\mathrm{P}} \sigma_{y}(\tau)$ can be clearly identifiable. Considering the same for the comparator we can expect that also ${ }^{\mathrm{C}} \sigma_{\mathrm{y}}(\tau)$ is well reproducible. The difficulty is with the additional variations that originate from sources such as unstable environment and electromagnetic interference that make up the contribution ${ }^{\mathrm{M}} \sigma_{\mathrm{y}}(\tau)$. These additional variations may occur irregularly thus making the short-term background process non-stationary i.e. depending on the time of its measurement. If periodic, they can distort the stability plot $\sigma_{\mathrm{y}}(\tau)$ in any region of $\tau$.

If we measure the same pair repeatedly, an $i$-th measurement series provides the short-term stability $\sigma_{\mathrm{y}}(\tau)_{\mathrm{i}}$ which can be thought of as a sample short-term stability of the long-term continuous process $\left\{x_{\mathrm{k}}\right\}$ whose stability is $\sigma_{\mathrm{y}}(\tau)$ for $N \rightarrow \infty$.

We can rewrite (2) for the $i$-th measurement series as

$$
\sigma_{\mathrm{y}}(\tau)_{\mathrm{i}}=\left[{ }^{\mathrm{P}} \sigma_{\mathrm{y}}(\tau)^{2}+{ }^{\mathrm{C}} \sigma_{\mathrm{y}}(\tau)^{2}+{ }^{\mathrm{M}} \sigma_{\mathrm{y}}(\tau)_{\mathrm{i}}{ }^{2}\right]^{1 / 2}
$$

based on the previous presumption that what may vary from one measurement to another is merely the contribution ${ }^{\mathrm{M}} \sigma_{\mathrm{y}}(\tau)$ while the inherent stabilities ${ }^{\mathrm{P}} \sigma_{\mathrm{y}}(\tau)$ and ${ }^{\mathrm{C}} \sigma_{\mathrm{y}}(\tau)$ remain unchanged.

We consider the process $\left\{x_{\mathrm{k}}\right\}$ stationary in the sense of "short-term stability" if $\sigma_{\mathrm{y}}(\tau)_{\mathrm{i}}, i=1,2, \ldots$ is equal within the error bars given by measurement statistics. Thus if we may presume that the process $\left\{x_{\mathrm{k}}\right\}$ is stationary, then repeated measurements may not seem necessary. In these highly sensitive measurements, however, this cannot be presumed. Moreover, the classical DMTDM requires that the measured signals be kept quasi synchronous in order to ensure the maximum rejection of $\mathrm{CO}$ noise, which with free running oscillators limits the length of measurement period.

\section{INSTRUMENTATION}

\section{A. Measured Oscillators}

As hinted previously, we had at our disposal four $5 \mathrm{MHz}$ OSA oscillators: two 8600-BC5GE with serial numbers 291 and 315 (further denoted as A and B) possessed by IPE, and two 8607-BM with serial numbers 102 and 199 (further denoted as C and D) possessed by OSA and FEMTO-ST, respectively. The A, B oscillators had been in continuous operation for more than a year before the measurement but the C, D oscillators for only 3.5 days. The C, D oscillators have the original casing while the A, B oscillators are housed in extra cases to improve the shielding and are also supplied with an arrangement for fine tuning with a resolution $<1 \times 10^{-12}$. The same tuning arrangement was temporarily supplied to C, D 
oscillators during the campaign. Each oscillator provides two sine-wave signals on SMA connectors with a power level of $+7 \mathrm{dBm}$.

\section{B. Time Domain}

1) Main Comparator: The main comparator has been developed at IPE in cooperation with LNE-SYRTE [16]. The system is based on the classical DMTDM operating at $5 \mathrm{MHz}$. The dual mixing to $v_{\mathrm{B}}=5 \mathrm{~Hz}$ provides the basic sampling interval $\tau_{0}=200 \mathrm{~ms}$ and $\mathrm{M}=10^{6}$. The system bandwidth is given by a single-pole low-pass filter with a corner frequency of $15 \mathrm{~Hz}$. The common oscillator (CO) used during the campaign was a $10 \mathrm{MHz} \mathrm{HP} 10811$ with a frequency divider providing $5 \mathrm{MHz}$ at $+11 \mathrm{dBm}$ power level with $\mathrm{L}(\mathrm{f})=-152 \mathrm{dBc} / \mathrm{Hz}$ of white phase modulation (WPM) noise. The time difference $x(t)$ from DMTDM was measured with a Stanford Research 620 time-interval counter. Further in this text it will be referred to as IPE1 comparator. Later on the IPE1 was reconstructed. The HP10811 CO was replaced with a $5 \mathrm{MHz}$ Milliren MTI260-504A followed by a low-noise amplifier providing a power level of $+11 \mathrm{dBm}$ with $\mathrm{L}(\mathrm{f})=-161 \mathrm{dBc} / \mathrm{Hz}$ of WPM. In addition, all BNC connectors within the DMTDM block have been replaced with SMA connectors and the flexible cables with semi-rigid cables. We will refer to this reconstructed version as IPE2 comparator.

The noise performance of IPE1 and IPE2 comparators has been tested with two signals at $+3 \mathrm{dBm}$ power-split from the $+7 \mathrm{dBm}$ output of a single BVA oscillator (in the IPE1 test from oscillator D and in the IPE2 test from oscillator B). This is a common test based on the presumption that for the test signals originating from a single source we have ${ }^{\mathrm{P}} \sigma_{\mathrm{y}}(\tau)<<{ }^{\mathrm{C}} \sigma_{\mathrm{y}}(\tau)$ and what we get from the test is $\sigma_{\mathrm{y}}(\tau)=\left[{ }^{\mathrm{C}} \sigma_{\mathrm{y}}(\tau)^{2}+{ }^{\mathrm{M}} \sigma_{\mathrm{y}}(\tau)^{2}\right]^{1 / 2}$. To ensure sufficient rejection of the $\mathrm{CO}$ noise, the test signals were set quasi-synchronous within $2 \times 10^{-3}$ of the beat-note period (the same synchronization interval was maintained during stability measurements). The results of the test are shown in Fig. 2. The increase in $\sigma_{\mathrm{y}}(\tau)$ at larger $\tau$ in this specific IPE1 test is owing to a periodic variation with a period of about $600 \mathrm{~s}$ whose origin is not known. Still there is no significant difference between IPE1 and IPE2 in the FFM region, i.e. in both cases the slope corresponds to flicker phase modulation (FPM) of $\sim 7 \times 10^{-15} / \tau$ which one would suppose can be neglected against the instability of the oscillator pair.

2) Supplementary Comparator: For supplementary measurements, we used a TSC 5110A Time Interval Analyzer serial number 129064 . The $5110 \mathrm{~A}$ analyzer provides the basic sampling interval $\tau_{0}=10 \mathrm{~ms}$ though our option generates the phase data only at $1 \mathrm{~s}$ intervals so the stabilities at $\tau<1 \mathrm{~s}$ are the screen shots. The multiplication factor is $5 \times 10^{6}$ at $5 \mathrm{MHz}$. The performance test has been made under similar conditions as that of the IPE1 and IPE2 comparators and its result is also depicted in Fig. 2 along with IPE1 and IPE2. Since $+3 \mathrm{dBm}$ is at the TSC specification limit $(+3 \mathrm{dBm}$ to $+17 \mathrm{dBm})$, we also tested it at a power level of $+9 \mathrm{dBm}$ by making use of a lownoise amplifier before the power splitter. The equivalent noise

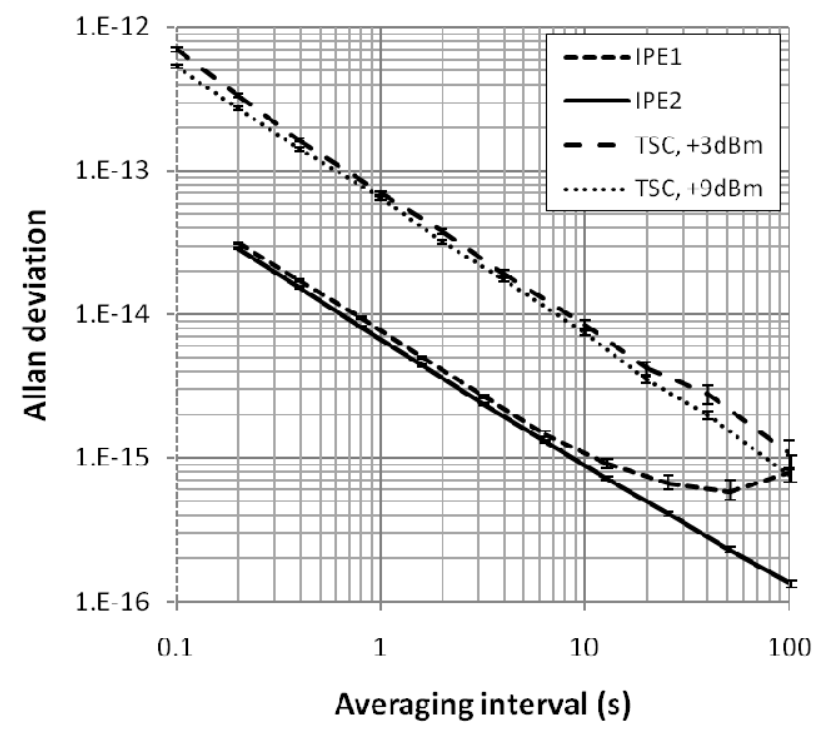

Figure 2. Performance test of IPE1/IPE2 comparators and 5110A.

bandwidth (BW) of TSC is about ten times larger than that of IPE. We have verified it by measuring a $5 \mathrm{MHz}$ signal with intentionally large WPM noise alternatively with IPE2 comparator and 5110A. The result was $\mathrm{BW}_{\mathrm{TSC}} / \mathrm{BW}_{\mathrm{IPE}}=\sigma_{\mathrm{y}}(\tau)_{\mathrm{TSC}}^{2} / \sigma_{\mathrm{y}}(\tau)_{\mathrm{IPE}}^{2}=10.3$ in the WPM region.

\section{Frequency Domain}

Complementary measurements in the frequency domain have been performed with a modified Femtosecond Systems FSS1000E Phase Noise Detector [17] connected to a SR760 FFT Spectrum Analyzer. We have modified the original FSS1000E because of its poorer noise performance (larger FPM probably due to the digital potentiometer at the FSS PLL input). We have by-passed the FSS PLL circuitry with a less noisy PLL of our own design.

\section{Laboratory Conditions}

The laboratory for stability measurement is housed in the underground vault which ensures a stable environment, especially concerning vibrations and mechanical shocks. The room is shielded, though far from perfect. The temperature is controlled to $23 \pm 1^{\circ} \mathrm{C}$. During the reported measurements the $24 \mathrm{~V}$ DC voltage for the BVA oscillators was supplied from two Statron 2229 double AC-DC sources with $2 \mathrm{mV}$ rms ripple (one Statron for $A \& B$ oscillators, the other for $C \& D$ oscillators). It should be noted that there was no other activity in the laboratory in addition to the measurement.

\section{PERFORMED MEASUREMENTS}

During the campaign with four oscillators we carried out 29 measurements with the IPE1 comparator and 18 supplementary measurements with 5110A. The four oscillators formed six pairs henceforth designated as A-B, A$\mathrm{C}$, etc. For the stability analysis we have used the following number of measurements (in parentheses) of respective pairs: A-B (4), A-C (4), A-D (4), B-C (5), B-D (6), C-D (6) made with IPE1, and A-B (2), A-C (2), A-D (2), B-C (2), B-D (2) and C-D (8) made with 5110A. The number of samples in one 
measurement series was typically 5000 . It should be noted that our measurement system did not allow us to measure more than one oscillator pair at the same time.

With the reconstructed IPE2 comparator, we have made around 50 additional measurements of the A-B pair in different periods of time.

\section{MEASUREMENT RESUlts}

\section{A. Measurement of A, B, C, D oscillators with IPEI}

Based on the IPE1 tests, we first assumed that the impact of the measurement system was negligible, i.e. $\sigma_{\mathrm{y}}(\tau) \approx^{\mathrm{P}} \sigma_{\mathrm{y}}(\tau)$, and that the individual variations were uncorrelated. Under this assumption we decomposed the pair stabilities into individual stabilities with the aid of the four-cornered hat technique using two different approaches.

In the analysis reported henceforth the phase data have first been checked for outliers (actually only a few outliers have been removed). The phase data used for stability calculation are residuals of the quadratic fit. The fit removal, however, has a negligible influence on the short-term results.

1) Least-square method: To decompose the six pair stabilities into four individual stabilities we have used the common least squares method applied to pair stabilities $\sigma_{\mathrm{y}}(\tau)_{\mathrm{i}}$ at all $\tau$ for all pairs. Denoting the pair stabilities as $\sigma_{\mathrm{AB}}(\tau)_{\mathrm{i}}$, $\sigma_{\mathrm{AC}}(\tau)_{\mathrm{i}}, \sigma_{\mathrm{AD}}(\tau)_{\mathrm{i}}, \sigma_{\mathrm{BC}}(\tau)_{\mathrm{i}}, \sigma_{\mathrm{BD}}(\tau)_{\mathrm{i}}$ and $\sigma_{\mathrm{CD}}(\tau)_{\mathrm{i}}$, and the individual stabilities $\sigma_{A}(\tau), \sigma_{B}(\tau), \sigma_{C}(\tau)$ and $\sigma_{D}(\tau)$, we can form a fourcornered hat as depicted in Fig. 3.

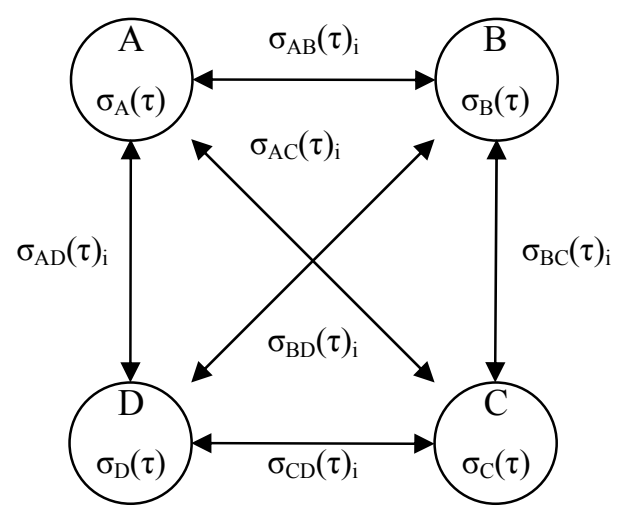

Figure 3. Four-cornered hat.

Based on this hat, we have for each $\tau$ an over-determined system of equations which can be solved for the individual stabilities $\sigma_{A}(\tau), \sigma_{B}(\tau), \sigma_{C}(\tau)$ and $\sigma_{D}(\tau)$. We can write the equations in the form

$$
\begin{aligned}
& \sigma_{\mathrm{AB}}^{2}(\tau)_{1}=\sigma_{\mathrm{A}}^{2}(\tau)+\sigma_{\mathrm{B}}^{2}(\tau)+\varepsilon_{\mathrm{AB} 1}^{2} \\
& \sigma_{\mathrm{AB}}^{2}(\tau)_{2}=\sigma_{\mathrm{A}}^{2}(\tau)+\sigma_{\mathrm{B}}^{2}(\tau)+\varepsilon_{\mathrm{AB} 2}^{2} \\
& \ddot{\sigma}^{2} \\
& \sigma_{\mathrm{AC}}(\tau)_{1}=\sigma_{\mathrm{A}}^{2}(\tau)+\sigma_{\mathrm{C}}^{2}(\tau)+\varepsilon_{\mathrm{AC} 1}^{2} \\
& \sigma_{\mathrm{AC}}^{2}(\tau)_{2}=\sigma_{\mathrm{A}}^{2}(\tau)+\sigma_{\mathrm{C}}^{2}(\tau)+\varepsilon_{\mathrm{AC} 2}^{2} \\
& \ldots \quad \text { etc. }
\end{aligned}
$$

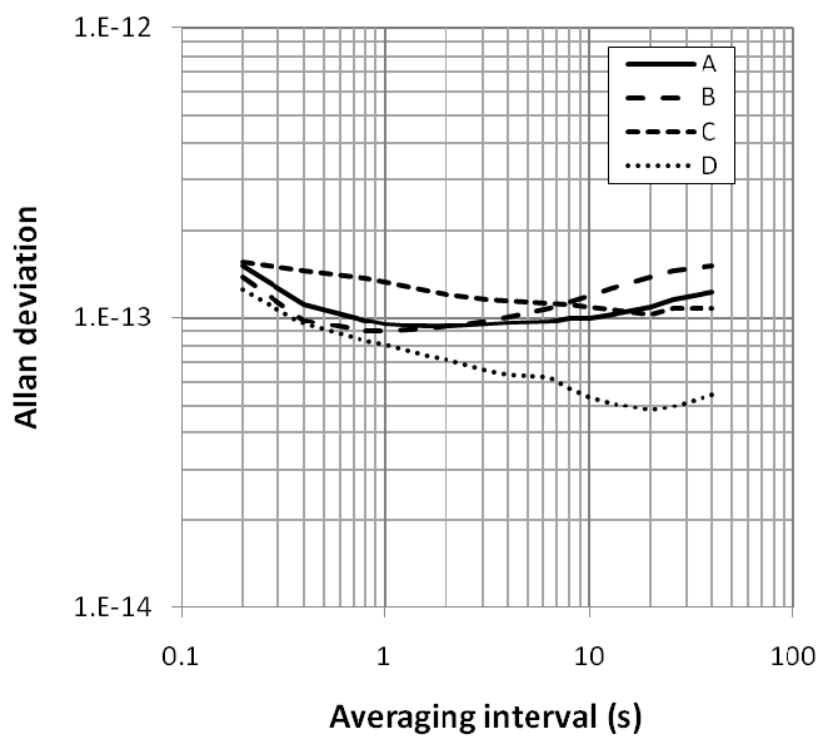

Figure 4. Individual stability from least squares decomposition.

The result of the solution is shown in the above Fig. 4. It should be noted that the use of weighted least squares has no impact on the FFM region.

2) Minimum FFM floor: This engineering approach is based on (2). In this case we are solely interested in the FFM noise which we consider the main goal of the time-domain short-term stability measurement of this type of oscillators. We assume that for a given pair any uncorrelated stochastic variations as well as periodic fluctuations make the amount of $\sigma_{\mathrm{y}}(\tau)_{\mathrm{i}}$ at FFM region of $\tau$ larger than the inherent FFM floor of ${ }^{\mathrm{P}} \sigma_{\mathrm{y}}(\tau)$. Thus the minimum of all $\sigma_{\mathrm{y}}(\tau)_{\mathrm{i}}, i=1,2, \ldots$ for the specific pair represents the best estimate of the inherent FFM floor. Clearly, we search for what we call a "justifiable" minimum which we define as $\sigma_{\mathrm{y}}(\tau)_{\mathrm{i}}$ at $\tau$ for which we have $\min \left[\sigma_{\mathrm{y}}(\tau)_{\mathrm{i}}+u(\tau)_{\mathrm{i}}, i=1,2, \ldots\right]$. We have searched the minima at $1 \mathrm{~s}<\tau<10 \mathrm{~s}$. The results based on this approach are shown in Table I.

TABLE I. FFM FLOOR ESTIMATED FROM JUSTIFIABLE MINIMUM.

\begin{tabular}{|c|c|c|c|c|}
\hline & $\mathrm{A}$ & $\mathrm{B}$ & $\mathrm{C}$ & $\mathrm{D}$ \\
\hline Allan deviation & $8.7 \times 10^{-14}$ & $9.4 \times 10-14$ & $1.1 \times 10-13$ & $6.0 \times 10-14$ \\
\hline
\end{tabular}

\section{B. Measurement of $A, B$ oscillators with IPE2}

Fig. 5 shows the stability plots of the A-B pair obtained from the measurements with the IPE1 comparator during the campaign (thin plots), from selected measurements made with the reconstructed IPE2 comparator at a later time (bold plots), and from selected measurements performed recently with 5110A (dashed plots). The error bars are omitted for sake of clarity. 


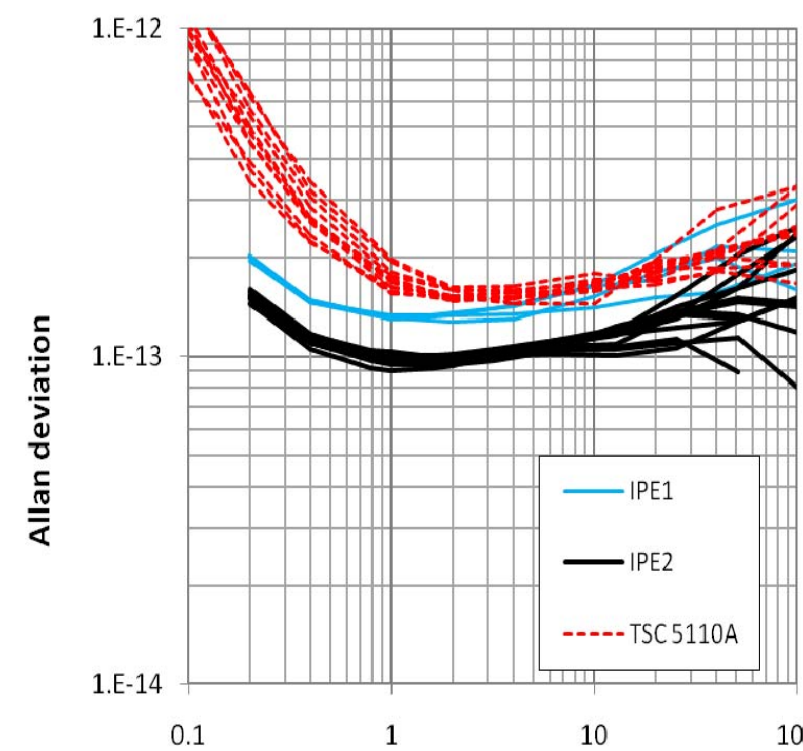

Averaging time (s)

Figure 5. Frequency stability of A-B pair.

The FFM floor is about $1.3 \times 10^{-13}$ with IPE1, $0.9 \times 10^{-13}$ with IPE2 and $1.5 \times 10^{-13}$ with $5110 \mathrm{~A}$. Thus a significant improvement has been observed after the reconstruction of the IPE comparator. A question arises about which of the contributions in (2) is responsible for this improvement. We can hardly expect that it has been due to improved inherent stability of the oscillators since in both measurements with IPE1 and IPE2, the oscillators had been continuously operating for a long time. Therefore we must attribute it to better performance of the measurement system, i.e. to smaller variance ${ }^{\mathrm{C}} \sigma_{\mathrm{y}}(\tau)^{2}+{ }^{\mathrm{M}} \sigma_{\mathrm{y}}(\tau)^{2}$. Since we are not aware of any changes that may have lead to significantly smaller ${ }^{\mathrm{M}} \sigma_{\mathrm{y}}(\tau)$, the responsible contribution is most likely the inherent instability of the comparator, ${ }^{\mathrm{C}} \sigma_{\mathrm{y}}(\tau)$. If this is true then the contribution to the total FFM of $1.3 \times 10^{-13}$ from the IPE1 comparator was as large as $9 \times 10^{-14}$. This is evidently in contradiction to what we would expect from the IPE1/IPE2 tests which differ little giving the FPM noise of $\sim 7 \times 10^{-15} / \tau$ in the short run as shown in Fig.2. This FPM noise is negligible against the expected FFM floor of the oscillators. This implies that the comparator performs differently with the signals from a single source (as in the test) and from two different sources (as in the stability measurement). We suspect that the $\mathrm{CO}$ noise is rejected differently in the two modes of measurement, based on the fact that the WPM noise in the CO used in the IPE1 comparator was about three times larger than that in the reconstructed IPE2. Actually we also suspect that this effect has manifested itself in a different manner depending on the oscillator pair. At the time of the writing this text we are not able to explain this effect.

As a result of this finding, the individual stabilities based on the measurement with IPE1 bear an unknown error because the pair stabilities that entered into the four-cornered hat were correlated. Provided that the A and B oscillators have approximately equal inherent FFM floor (which can be deduced from the A-D and B-D pairs), the A-B pair floor of $9 \times 10^{-14}$ found with IPE2 gives the individual floor of $A$ and $B$ at a level of $6 \times 10^{-14}$ (neglecting the contribution from IPE2 itself) which is considerably less than what we have obtained with IPE1.

\section{Other Observations}

1) Non-stationary background: In several measurements performed during the campaign, we have observed nonstationary disturbing processes. Namely, at some $\tau$ the values of $\sigma_{\mathrm{y}}(\tau)_{\mathrm{r}} \pm u_{\mathrm{r}}(\tau)$ and $\sigma_{\mathrm{y}}(\tau)_{\mathrm{s}} \pm u_{\mathrm{s}}(\tau)$, resulting from the measurement series $r$ and $s$, respectively, have given the difference $\left|\sigma_{\mathrm{y}}(\tau)_{\mathrm{r}}-\sigma_{\mathrm{y}}(\tau)_{\mathrm{s}}\right|>u_{\mathrm{r}}(\tau)+u_{\mathrm{s}}(\tau)$ even with the uncertainties extended to $\pm 3 \sigma$ (99\% probability).

2) Disturbing periodicity: Fig. 6 shows an uncommon distortion of the stability plot observed in an A-D measurement series. Here the FFM floor is distorted by two periodic processes which manifest themselves in different intensity in the course of the measurement series. This effect has been detected by cutting the data into two sections. A Stable32 routine called Dynamic Stability [18], [19] is a good tool to analyze this kind of phenomena. The minimum of $\sim 1 \times 10^{-13}$ from the first half of data (of which, however, a significant contribution originates from the IPE1 comparator) approximates the FFM floor of the A-D pair better than the minimum from the complete data.

3) Dispersion among measurement series: In the measurements with IPE1, the standard deviation of $\sigma_{\mathrm{y}}(\tau)_{\mathrm{i}}$ at $\tau=1 \mathrm{~s}$ is within the range $1.7 \times 10^{-15}$ (pair A-D) to $5.7 \times 10^{-15}$ (pair C-D). The number of measurement series is too small to draw any conclusion from this observation.

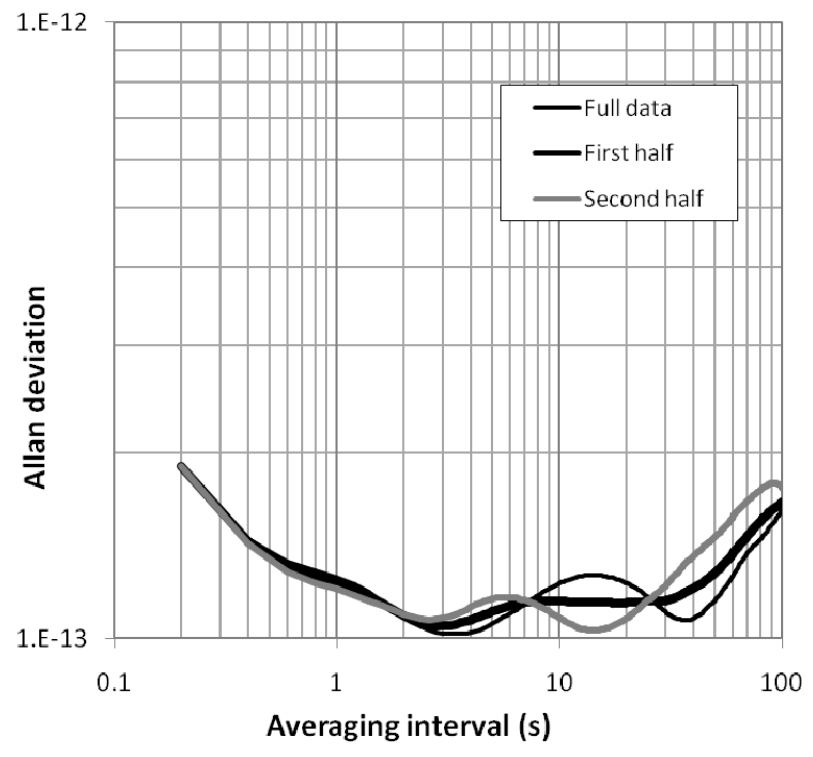

Figure 6. Illustration of disturbing periodicity. 
4) False phase locks: When measuring free running highly-stable oscillators which are detuned in the order of $10^{-13}$ and which are interconnected through the measurement system, there is always a risk for the oscillators of being pulled-in. This may end up in a false lock or just in disturbing the free-running phase. The false lock is clearly identifiable from the phase plot but it is difficult to detect a disturbing pull-in process. There are basically two ways to induce the pull-in process in these oscillators: by a reverse signal injection or through the varactor diode. Fortunately these effects are much reduced thanks to OSA's careful design. Close inspection of the phase data of all measurement series has not revealed any false phase locks. The pull-in processes, if there are any, could not be discerned from normal noise variations.

\section{CONCLUSIONS}

The measurements of the four oscillators have shown that the background instability of the comparators used, i.e. IPE1 and $5110 \mathrm{~A}$, was too large to be neglected. This has been found in later measurements of the two of the four oscillators performed with the reconstructed IPE2 comparator which provided better results than IPE1. Thus the results from fourcornered hat shown in Fig. 4 bear unknown errors. Therefore we plan to repeat the measurement of three oscillators (A, B and D) with the IPE2 comparator in order to determine the individual stabilities more accurately. We can conclude though that the two IPE oscillators (A and B) have the inherent FFM floor at a level of $6 \times 10^{-14}$ and that the FEMTOST oscillator (D) even has the inherent FFM floor $<6 \times 10^{-14}$.

Our current knowledge does not allow us to explain the finding that the DMTDM comparators perform differently in the test (with signals from a single source) and in the frequency stability measurement (with signals from different sources). This effect will be further investigated. For now it is imperative for us to consider this test as "the necessary but not sufficient" condition for a good comparator performance with uncorrelated signals. The key problem of course is that we have no less noisy uncorrelated signals to test the comparator.

It should also be noted that only recently we have found that much of the IPE1 comparator inherent instability was due to mismatches of the frequency divider used after HP10811 $\mathrm{CO}$ (see B 1).

\section{ACKNOWLEDGMENT}

The Czech authors thank Dr. Roland Barillet of LNESYRTE for his help in the development of the IPE DMTDM comparator.

\section{REFERENCES}

[1] J.-P. Aubry, J. Chauvin, and F. Sthal, "A new generation of very high stability of BVA oscillators," Proc. $21^{\text {st }}$ EFTF- IEEE FCS, June 2007, in press.

[2] http://www.oscilloquartz.com/file/pdf/8600.pdf

[3] http://www.oscilloquartz.com/file/pdf/8607.pdf

[4] D.W. Allan and H. Daams, "Picosecond time difference measurement system," Proc. 29th Annu. Symp. Frequency Contr., Atlantic City, USA, pp. 404-411, 1975.

[5] S. Stein, D. Glaze, J. Levine, J. Gray, D. Hilliard, D. Howe and L.A. Erb, "Automated high-accuracy phase measurement system," IEEE Trans. Instrum. Meas. vol. IM-32, pp. 227-231, (1983).

[6] S.R. Stein, "Frequency and time - their measurement and characterization," in Precision Frequency Control, vol. II, E.A. Gerber and A. Ballato, Eds. New York: Academic Press, pp.229-231, (1985).

[7] L. Sze-Ming, "Influence of noise of common oscillator in dual-mixer time-difference measurement system", IEEE Trans. on Instr. and Meas., vol. IM-35, pp. 648-651 (1986).

[8] R. Barillet, "Ultra-low noise phase comparator for future frequency standards (Comparateur de phase ultra faible bruit pour les futurs étalons de frequence)," Proc 3th EFTF, pp. 249-254, March 1989.

[9] C.A. Greenhall, "Common-source phase error of a dual-mixer stability analyzer," TMO Progress Report 42-143, Jet Propulsion Laboratory, November 2000.

[10] G. Brida, "High resolution frequency stability measurement," Rev. Sci. Instrum., vol. 73, pp. 2171-2174, May 2002.

[11] L. Šojdr, J. Čermák and G. Brida. "Comparison of high-precision frequency-stability measurement systems," Proc. Joint IEEE FCS/EFTF Meeting, pp. 317-325, May 2003.

[12] L. Šojdr, J. Čermák and R. Barillet, "Optimization of dual-mixer time-difference multiplier," Proc. 18th EFTF, CD: Session 6B/130.pdf, April 2004.

[13] D.W. Allan, "The statistics of atomic frequency standards," Proc. IEEE, vol. 54, No. 2, pp. 221-230, February 1966.

[14] J.A. Barnes, et al, "Characterization of frequency stability," IEEE Trans. Instrum. Meas., Vol. IM-20, No. 2, pp. 105-120, May 1971.

[15] "Stable32 version 1.35: frequency stability analysis," Hamilton Technical Services, S. Hamilton, MA 01982 USA, (2002).

[16] W.J. Riley, "Confidence intervals and bias corrections for the Stable32 variance functions", Hamilton Technical Services, 2000.

[17] "FSS1000E Phase noise detector," Operation Manual Rev 3.2, Femtosecond Systems, Inc., pp.4-6, (1999).

[18] L. Galleani and P. Tavella, "The characterization of clock behavior with the dynamic Allan variance," Proc. Joint IEEE FCS/EFTF Meeting, pp. 239-244, May 2003.

[19] L. Galleani and P. Tavella, "Tracking nonstationarities in clock noises using the dynamic Allan variance," Proc. Joint IEEE FCS/PTTI Meeting, (2005). 